BONTHRON EDITED

ORIGINAL ARTICLE

\title{
High-grade serous carcinomas arise in the mouse oviduct via defects linked to the human disease
}

Yali Zhai $^{\mathrm{a}}$, Rong Wu ${ }^{\mathrm{a}}$, Rork Kuick ${ }^{\mathrm{b}}$, Michael S. Sessine ${ }^{\mathrm{a}}$, Stephanie Schulman ${ }^{\mathrm{a}}$, Megan Green $^{\mathrm{a}}$, Eric R. Fearon ${ }^{\mathrm{a}, \mathrm{c}, \mathrm{d}}$, and Kathleen R. Cho ${ }^{\mathrm{a}, \mathrm{c}}$

Departments of Pathology ${ }^{a}$, Internal Medicine ${ }^{c}$ and Human Genetics ${ }^{d}$, University of Michigan Medical School, 1506 BSRB, 109 Zina Pitcher Place, Ann Arbor, MI 48109, USA; Department of Biostatistics $^{b}$, University of Michigan School of Public Health, M4509 SPH II, 1415 Washington Heights, Ann Arbor, MI 48109, USA

\section{Running Title:}

Mouse models of oviductal high-grade serous carcinoma

\section{Corresponding Author:}

Kathleen R. Cho, 1506 BSRB, 109 Zina Pitcher Place, University of Michigan, Ann Arbor, MI 48109-2200, USA; email: kathcho@umich.edu

\section{Conflicts of Interest:}

The authors declare no potential conflicts of interest

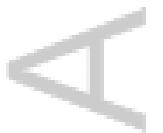

This is the author manuscript accepted for publication and has undergone full peer review but has not been through the copyediting, typesetting, pagination and proofreading process, which may lead to differences between this version and the Version of Record. Please cite this article as doi: $10.1002 /$ path.4927

This article is protected by copyright. All rights reserved. 


\section{Abstract}

Recent studies suggest that the most common and lethal type of "ovarian" cancer, high-grade serous carcinoma (HGSC), usually arises from epithelium on the fallopian tube fimbriae, and not from the ovarian surface epithelium (OSE). We have developed Ovgp1-iCreER ${ }^{T 2}$ mice in which the Ovgp1 promoter controls expression of tamoxifen (TAM)-regulated Cre recombinase in oviductal epithelium - the murine equivalent of human fallopian tube epithelium (FTE). We employed Ovgp1-iCreER ${ }^{T 2}$ mice to show that FTE-specific inactivation of several different combinations of tumour suppressor genes recurrently mutated in human HGSCs - namely Brca1, Trp53, Rb1, and Nf1 - results in serous tubal intraepithelial carcinomas (STICs) that progress to HGSC or carcinosarcoma, and to widely metastatic disease in a subset of mice. The cancer phenotype is highly penetrant and more rapid in mice carrying engineered alleles of all four tumour suppressor genes. Brca1, Trp53 and Pten inactivation in the oviduct also results in STICs and HGSCs, and is associated with diffuse epithelial hyperplasia and mucinous metaplasia not observed in mice with intact Pten. Oviductal tumours arise earlier in these mice, compared to those with Brca1, Trp53, Rb1 and Nf1 inactivation. Tumour initiation and/or progression in mice lacking conditional Pten alleles likely requires acquisition of additional defects, a notion supported by our identification of loss of the wild-type $R b 1$ allele in the tumours of mice carrying only one floxed $R b 1$ allele. Collectively, the models closely recapitulate the heterogeneity and histological, genetic, and biological features of human HGSC. These models should prove useful for studying the pathobiology and genetics of HGSC in vivo, and for testing new approaches for prevention, early detection, and treatment.

Keywords: ovarian cancer, genetically engineered mouse model, fallopian tube, serous carcinoma 


\section{Introduction}

High-grade serous carcinoma (HGSC) is the most common and lethal type of "ovarian" cancer, accounting for $\sim 70-75 \%$ of cases [1]. Recent studies suggest that HGSCs usually arise in fallopian tube epithelium (FTE), rather than the ovarian surface epithelium (OSE) [2,3]. HGSCs display a high level of chromosomal instability, and virtually all harbour somatic TP53 mutations, which occur very early in HGSC pathogenesis, as TP53 mutations are also present in HGSC precursors, known as serous tubal intraepithelial carcinomas (STICs) [4-6]. Although only nine genes were shown to be significantly mutated in HGSCs by the Cancer Genome Atlas project [7], somatic structural alterations (e.g. DNA copy number alterations, gene breakage, gene fusions) are frequently seen in these tumours $[7,8]$. These mutational and structural changes often lead to dysfunction of the RB, BRCA, PI3K and RAS pathways. Genetic instability in the early lesions presumably contributes to the likelihood that somatic mutations conferring metastatic potential will be acquired. Thus, women with HGSCs typically have small primary lesions and widespread metastases at diagnosis. Ovarian carcinosarcomas (also known as malignant mixed Müllerian tumours - MMMTs) show both malignant epithelial and mesenchymal elements, and are now thought to represent a much less common variant of HGSC [9]. Like HGSCs, pelvic/ovarian carcinosarcomas have been associated with STICs, and a recent study showed identical TP53 mutations in matched pairs of STIC and carcinosarcoma, providing evidence of their clonal relationship and the potential origin of carcinosarcoma in the fallopian tube $[10,11]$.

Genetically engineered mouse models (GEMMs) of ovarian cancer that closely recapitulate their human tumour counterparts provide excellent in vivo systems with which to study tumour biology, and to perform pre-clinical studies aimed at improving ovarian cancer prevention, early detection, and therapy [12]. Historically, most HGSC GEMMs were based on OSE transformation [13-16]. More recently, models based on transformation of oviduct (equivalent to human fallopian tube) epithelium (hereafter referred to as FTE) have been reported. Kim et al. developed an oviductal HGSC model based on conditional deletion of Dicer and Pten, using the Amhr2 promoter to express Cre recombinase in the murine oviduct [17]. The tumours in this 
model arise first in the fallopian tube stroma and are initially mesenchymal. More recently, Perets et al. described a HGSC GEMM, using the Pax8 promoter to drive Cre-mediated recombination of floxed Brca1 or Brca2, Pten, and Trp53 alleles in the FTE of Pax8-rtTA;TetOCre mice [18]. While these oviductal HGSC models represent a significant advance, both direct Cre expression using gene promoters that lack specificity for the FTE. Amhr2 is also expressed in the OSE and stromal cells in the ovary, oviduct, and other portions of the female genital tract [19], while Pax8 is expressed in other Müllerian epithelia, such as endometrial glandular epithelium, as well as other sites such as kidney and thyroid [20]. A promoter for directing Cre expression to the FTE with greater specificity was highlighted by Miyoshi et al., who reported the development of oviductal HGSC-like tumours in mice expressing SV40-LTag under control of a 2.2-kb fragment of the murine Ovgp1 (oviductal glycoprotein1) promoter [21]. We have developed mice in which the Ovgp1 promoter directs expression of tamoxifen (TAM)-regulated Cre recombinase in the FTE [22]. Ovgp1-iCreER ${ }^{T 2}$ mice carrying floxed alleles of the Apc and Pten tumour suppressor genes (TSGs) invariably develop endometrioid-like tumours in the FTE following treatment with TAM, and these tumours are more similar to human ovarian endometrioid carcinomas in their morphology, biological behavior, and gene expression profiles than tumours based on Apc and Pten inactivation in the OSE [22]. While our earlier work showed that cell of origin has a profound impact on tumour phenotype, in the present study we wished to test the hypothesis that genetics also affects tumour phenotype. Given that dysregulation of Wnt and PI3K/AKT signalling - characteristic of human endometrioid carcinomas - in the oviductal epithelium results in mouse tumours with endometrioid-like morphology, we wanted to test whether inactivation of TSGs that are often mutated in human HGSCs (Brca1, Trp53, Rb1, and Nf1) [7], lead to HGSC-like tumours in the mouse oviduct. To build on the work of Perets et al., we also wished to test the effects of Brca1, Trp53, and Pten inactivation in our model system [18]. Although somatic PTEN point mutations are relatively uncommon in HGSCs, dysregulation of the PI3K/AKT signalling pathway is frequently observed, often as a consequence of PTEN gene breakage events or PIK3CA amplification $[7,8]$. 


\section{Materials and methods}

\section{Genetically modified mice and animal care}

The development and characterization of Ovgp1-iCreER $R^{T 2}$ transgenic mice have been described in detail [22]. Ovgp1-iCreER ${ }^{T 2}$ transgenic mice were crossed with mice carrying engineered Rb1, Trp53, Brca1, Nf1, and Pten alleles, to generate transgenic mice with various combinations of TSG alterations. In addition to floxed Rb1, Trp53, Brca1, Nf1, and Pten alleles in which Cre-mediated recombination generates null alleles, we employed mice carrying a Creinducible Trp53 mutation (LSL-R172H) and mice with constitutional inactivation of one Brca1 allele $\left(B r c a 1^{d e l}\right) . R b 1^{f l}, \operatorname{Trp} 53^{f l}, \operatorname{Trp} 53^{L S L-R 172 H}$, and $B r c a 1^{f l}$ mice were obtained from the National Cancer Institute's mouse repository. $N f 1^{f / f l}$ mice were a gift from Yuan Zhu (currently at Children's National Medical Center, Washington, DC, USA). Pten $n^{f / f l}$ mice were a gift from Tak Mak (University Health Network, Toronto, Ontario, Canada). Mice with the Brca ${ }^{\text {del }}$ allele were generated by crossing E2a-Cre mice (B6.FVB-Tg[Ella-cre]C5379Lmgd/J, Jackson Laboratory, Bar Harbor, Maine, USA) with $B r c a 1^{f /+}$ mice to generate mice carrying an inactivated Brca1 allele in the germline. All strains were maintained on a mixed genetic background. Mouse genotypes were confirmed by PCR analysis of tail DNA (primer sequences in Supplementary Table S1). Procedures involving mice were approved by the University of Michigan's Institutional Animal Care and Use Committee (PRO00006370).

\section{In vivo induction of oviductal tumours}

Ovgp 1-iCre-ER $R^{T 2}$ female mice carrying various engineered TSG alleles were given intraperitoneal injections of tamoxifen (TAM, T5648, Sigma-Aldrich, Indianapolis, IN, USA) at $100 \mathrm{mg} / \mathrm{kg}$ of body weight on three consecutive days, usually when mice were 6 to 8 weeks old. Initially, while monitoring mice for tumour development, selected mice were euthanized at various time points following TAM injection and inspected for tumour location and extent at necropsy. Thirteen mice underwent survival surgery, during which the right oviduct and ovary were removed at two $(n=11)$, three $(n=1)$ or twelve $(n=1)$ months after TAM, and the mice were then monitored for tumour development in the remaining oviduct. Once the presence of a 
tumour was established, most mice were monitored to humane endpoints. Mice were grossly evaluated for tumour extent, and in each case, ovaries, oviducts, uteri, lungs, multiple abdominal organs, omentum, and mesentery were examined microscopically.

\section{Histopathology and immunohistochemistry}

Tissues were fixed in $10 \%$ buffered formalin and paraffin-embedded. Sections $(5 \mu \mathrm{m})$ were H\&E-stained for evaluation by light microscopy. To identify microscopic oviductal lesions, each oviduct and ovary were serially sectioned in their entirety for microscopic examination. Alternate sections were either stained with H\&E or retained for immunohistochemical (IHC) staining, performed using standard methods as previously described [22]. Antigen retrieval was performed by microwaving the slides in citrate buffer, pH 6.0 (Biogenex, San Ramon, CA, USA) for $15 \mathrm{~min}$. Antigen-antibody complexes were detected using the avidin-biotin peroxidase method, with diaminobenzidine (DAB) as the chromogenic substrate (Vectastain ABC kit, Vector Laboratories, Burlingame, CA, USA). Primary antibodies used were: rat anti-cytokeratin 8 (Developmental Studies Hybridoma Bank, University of lowa), rabbit anti-p53 and rabbit antiPAX8 (Proteintech, Chicago, IL, USA), rat anti-Ki67 (DAKO, Carpinteria, CA, USA) and goat anti-OVGP1 (Santa Cruz Biotechnology, Inc., Santa Cruz, CA, USA).

\section{Results}

\section{STICs, HGSCs, and MMMTs develop in the mouse oviduct following conditional inactivation of various combinations of Rb1, Brca1, Trp53 and/or Nf1 in the FTE}

We crossed Ovgp1-iCreER ${ }^{T 2}$ mice with mice carrying Brca1, Trp53, Rb1, and Nf1 alleles, to generate 80 transgenic mice of several different genotypes (Figure 1 and Supplementary Table S2). Mice with conditionally modified Brca1, Trp53 and Rb1 alleles are hereafter referred to collectively as BPR mice $(\mathrm{n}=29)$. BPN mice $(\mathrm{n}=3)$ are those with modified Brca1, Trp53 and Nf1 alleles, while BPRN mice $(n=48)$ carry modified alleles for all four TSGs. Data summarizing the 
oviductal lesions developing in these mice are shown in Figure 1, in which animals with generally similar genotypes are grouped together.

In order to determine whether Brca1, Trp53, Rb1, and/or Nf1 inactivation in the FTE results in an oviductal tumour phenotype, ten mice with a range of defined genotypes were euthanized 1.5 to 6.5 months after TAM. One BPRN mouse (\#2820) showed a microscopic lesion closely resembling human STIC in one oviduct 2.5 months after TAM injection. Oviductal lesions were not identified in the remaining nine mice. In an attempt to reduce the number of mice needed to determine if, and how often, mice with different genotypes acquire oviductal tumours, the right oviduct and ovary were removed from thirteen mice in a survival surgery procedure. Eleven of the thirteen (four BPRN, six BPR, and one BPN) had the right oviduct and ovary removed two months after TAM injection. STIC was found in one BPRN mouse (\#3867, with homozygous floxed alleles for all four TSGs). Two additional BPRN mice underwent survival surgery at 3 and 12 months after TAM (\#3559 and \#8234), and STIC was identified in the latter.

Photomicrographs of two representative mouse STIC lesions are shown in Figure 2. As in humans, the mouse STICs have a predilection for arising in the distal portion of the oviduct (Figures $2 \mathrm{~A}$ and $2 \mathrm{C}$, equivalent to human fallopian tube fimbriae). The lesional cells display enlarged, hyperchromatic and pleomorphic nuclei, prominent nucleoli, increased mitotic activity, and loss of polarization (Figures 2B and 2D).

All thirteen mice that underwent survival surgery were monitored for tumour development in the remaining oviduct for 5 to 23 months following TAM injection. Lesions were identified in the remaining oviduct in five of six BPRN mice, two of six BPR mice, and in the BPN mouse. The oviductal lesions identified in the remaining oviduct included two STICs, two early HGSCs (confined to oviduct), two HGSCs extending beyond the oviduct, and two MMMTs, one of which metastasized widely.

Oviductal lesions (STIC, early HGSC, HGSC and/or MMMT) were identified in 50 of 57 additional mice (12 BPR, $36 B P R N$, and $2 B P N$ mice) not included in the groups described above. These mice were monitored for tumour development for time periods ranging from 7 to 24 months after TAM (Figure 1). In the 59 of 80 mice with oviductal lesions, tumour involved 
one or both ovaries in 24 (41\%), obliterating the ovary in all but four cases. Widespread peritoneal metastases were observed in 8 mice (14\%) and ascites in 7 (12\%). In similar fashion to the human disease, metastases were often observed in the omentum. Representative photomicrographs showing tumour progression from STIC to early HGSC, HGSC/MMMT, and metastatic disease are shown in Figure 3 , and IHC staining of primary tumours at various stages of tumour progression, and metastases to omentum and ovary are shown in Figure 4 and Supplementary Figure S1, respectively. The tumour cells expressed CK8 and PAX8 and showed increased proliferation, based on IHC staining for Ki67. Tumours with the conditional Trp53 R172H mutant allele only occasionally showed focal stabilization of p53 protein (Figure 4, \#3724). While the neoplastic cells in STICs and HGSCs retain expression of PAX8, a marker of secretory cells, they do not express tubulin, which marks ciliated cells (Supplementary Figure S2). The absence of tubulin expression is not surprising, as human HGSCs and STICs do not typically display ciliated morphology.

Only 6 of $48(12.5 \%) B P R N$ mice, compared to 15 of $29(52 \%) B P R$, failed to develop lesions. Some of the failures are likely attributable to euthanasia at relatively early time points ( $\leq 6.5$ months after TAM), particularly in the BPR mice. All three BPN mice included in the study developed oviductal HGSC and/or MMMT. On a per oviduct basis, and excluding those removed at the time of survival surgery or evaluated earlier than 7 months after TAM, 87 oviducts had at least one floxed $N f 1$ allele. In these oviducts, $11 \%$ had no neoplastic lesions, $21 \%$ had STIC, $25 \%$ had early HGSC, and 43\% had HGSC/MMMT. In contrast, for the 39 oviducts without floxed $N f 1$, the percentages were $36 \%, 41 \%, 13 \%$ and $10 \%$, respectively $(\mathrm{p}=$ $3.8 \times 10^{-5}$, chi-squared test; $p=2.7 \times 10^{-6}$, Mantel-Haenszel chi-squared test of association). Based on our analysis of the data shown in Figure 1 and in Supplementary Table S2, we conclude that the HGSC phenotype in our model is highly penetrant in BPRN and BPN mice, especially those carrying homozygous floxed alleles for all TSGs. Furthermore, disease progression is generally more rapid in BPRN than in BPR or BPN mice.

Cre-mediated recombination of Brca1, Trp53, Rb1 and Nf1 alleles in representative tumours was confirmed by PCR (Supplementary Figure S3). Interestingly, in mice carrying one floxed 
and one wild type $R b 1$ allele, loss of the wild type $R b 1$ allele was seen in seven of eight tumours analyzed (e.g. Supplementary Figure S3, \#s 13085, 13576, 13179, and 2117). Hence, as in human HGSC pathogenesis, the mouse tumours acquire additional genetic alterations during tumour progression. We evaluated oviductal tumour DNA for activation of the conditional $\operatorname{Trp53}{ }^{L S L-R 172 H}$ mutant allele in three representative tumours, two of which are shown in Supplementary Figure S3C. In all three tumours, recombination of the conditional mutant Trp53 allele was not observed. Interestingly, of mice with both oviducts intact for 7 months or more, 3 of 6 with the $T r p 53^{L S L-R 172 H /+}$ genotype had no detectable oviductal lesions, while only 4 of 51 mice with at least one floxed Trp53 allele lacked lesions $(p=0.020$, two-sided Fisher's exact test). These findings suggest that in this HGSC model system (i) there is selection for biallelic inactivation of $R b 1$ and (ii) the Trp53 $3^{f l}$ allele, generating a null Trp53 mutation via Crerecombination, is preferable to the $\operatorname{Trp} 53^{L S L-R 172 H}$ mutant allele, perhaps related to inefficient Cre recombination or to the absence of selective pressure for recombination and/or selection against recombination. Of note, in the absence of Cre-mediated activation of the $\mathrm{R} 172 \mathrm{H}$ mutation, the Trp53 ${ }^{L S L-R 172 H}$ allele is null [23].

In addition to the oviductal tumours, non-Müllerian tumours (e.g. lymphomas, soft tissue sarcomas, and adenomas/carcinomas of the thyroid, lung, skin, or breast) were identified in a subset of mice. Lymphomas were the most common type of non-oviductal tumour (17/80 $21 \%$ ). Interestingly, three of five mammary carcinomas arose in mice carrying one constitutional inactivated Brca1 allele. Development of lymphomas, sarcomas, carcinomas and other tumour types has been reported in various mouse strains, including some with high incidence [24-26]. Hence, development of non-oviductal tumours is not unexpected, given the advanced age of many of the mice and the presence of mutant Brca1 and/or Trp53 alleles in the germline of a sizeable subset. In order to determine if Cre-mediated recombination occurred in the nonMüllerian tumour cells, we evaluated DNA from representative tumours for the presence of recombined Brca1, Trp53, Rb1, and Nf1 alleles (Supplementary Figure S4). We found Cremediated recombination of engineered TSG alleles in one endometrial carcinoma (not shown) and in the presumptive thyroid carcinomas, which had morphological features reminiscent of human medullary carcinoma. The remaining tumours appear to arise spontaneously. We do not 
observe OVGP1 protein expression in the thyroid based on IHC staining, and hence allelic recombination in this site appears to be independent of endogenous Ovgp1 expression.

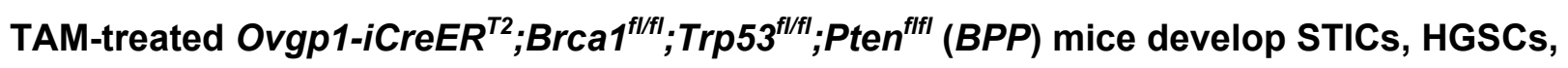
and MMMTs, and also display oviductal epithelial hyperplasia and mucinous metaplasia

We crossed Ovgp1-iCreER ${ }^{T 2}$ mice with animals carrying floxed Brca1, Trp53, and Pten alleles,

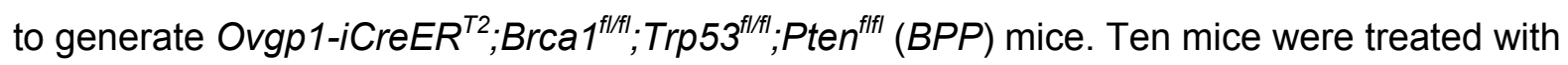
TAM, and two were euthanized at each of five time points (1, 2, 3, 4, and 6 months after TAM). STICs or early HGSC-like lesions were already present in treated mice one month after TAM. All ten mice developed bilateral oviductal lesions, including oviductal carcinomas at the later time points. One BPP mouse, examined 6 months after TAM, developed carcinosarcoma with metastasis to the ovary and ascites. Endometrial hyperplasia near the junction of the uterine horn with oviduct was noted in two of the ten BPP mice. Data from the BPP mice are summarized in Supplementary Table S3, and representative photomicrographs of H\&E and immunostained lesions arising in the oviducts of $B P P$ mice at 1 month, 2 months, and 6 months after TAM are shown in Figure 5. Like tumours arising in $B P R, B P R N$, and BPN mice, oviductal STICs (Figure 5A and B), HGSCs (Figure 5E, F, I, and J) and MMMTs (Figure 5M and N) arising in BPP mice express CK8 (Figure 5C, G, K and O) and PAX8 (Figure 5D, H, L, and P). As expected, they lack expression of PTEN and have an increased Ki-67 proliferative index (not shown). In addition, the oviducts of the TAM-treated BPP mice show rather diffuse epithelial hyperplasia characterized by areas with increased epithelial stratification, as well as mucinous metaplasia (Figure 6) in which the cells display prominent cytoplasmic accumulation of both acidic (Alcian blue-positive) and neutral (periodic acid Schiff-positive) mucin compared to normal oviductal epithelium. These latter findings were not observed in the BPR, BPN or BPRN mice. None of the BPP mice developed non-oviductal malignancies during the 6-month surveillance period.

\section{Discussion}


GEMMs that closely mimic the origin, genetics, and biological behaviour of HGSC would potentially be of great utility for testing strategies to improve HGSC prevention, early diagnosis, and treatment. Here, we have shown that Ovgp1-iCreER ${ }^{T 2}$ mice carrying conditional mutant TSG alleles relevant to human HGSC pathogenesis develop oviductal STICs, HGSCs, and carcinosarcomas that frequently metastasize to the ovaries and/or peritoneum and other organs. Although this is not the first GEMM to target the clinically relevant FTE, it does so with greater specificity than previously described models employing the Pax8 or Amhr2 promoters to direct expression of Cre recombinase in the oviductal epithelium.

We have previously shown that biallelic inactivation of $A p c$ and Pten leads to very different tumour phenotypes when induced in the OSE vs. the FTE, with the oviductal tumours closely resembling human endometrioid carcinomas, while the ovarian tumours were more poorly differentiated [22]. In other words, cell of origin plays an important role in determining tumour phenotype. We now show that different combinations of genetic alterations in the FTE also lead to very different tumour phenotypes. In short, while genetic alterations (e.g. Apc and Pten inactivation) that dysregulate signalling pathways characteristic of human endometrioid carcinomas result in endometrioid-like tumours in the mouse FTE [22], mutations commonly seen in human HGSCs result in HGSC-like oviductal tumours. Furthermore, although $B P R$, $B P R N, B P N$, and BPP mice all develop oviductal STICs and HGSCs/MMMTs following treatment with TAM, the timing of tumour development and progression varies significantly among the different models, and the lesions are not morphologically identical. Specifically, tumour development is very rapid and penetrant in BPP mice and the FTE acquires diffuse changes (hyperplasia and mucinous metaplasia) not observed in $B P R, B P N$, or BPRN animals. The short latency and rapid disease progression in BPP mice bear some similarity to our prior findings that mice rapidly develop oviductal endometrioid-like carcinomas following biallelic Apc and Pten inactivation by the Ovgp1-iCreER ${ }^{T 2}$ transgene [22]. In BPP and Apc ${ }^{\text {fl/fl}} ; P$ ten ${ }^{f / f l}$ mice, the rapidity of tumour development suggests that the conditional alterations are sufficient for neoplastic transformation of the targeted cells. In contrast, in BPR, BPN, and BPRN mice, even those with homozygous floxed alleles for all TSGs, oviductal tumours take longer to develop and progress. Progression from STIC to HGSC/MMMT usually takes well over a year in BPR mice, 
but is accelerated by addition of biallelic $N f 1$ inactivation. These findings suggest that tumour initiation and/or progression in BPR, BPN, and BPRN mice probably requires acquisition of additional defects, a notion supported by our identification of loss of the wild-type $R b 1$ alleles in the tumours of mice carrying only one floxed $R b 1$ allele. The relatively long latency and presumptive need for additional genetic alterations may actually be considered advantageous features of the model system, as the resultant tumour heterogeneity may more closely mimic HGSC pathogenesis in humans than the rapid neoplastic transformation of FTE observed in the context of Apc/Pten or Brca1/Trp53/Pten inactivation. Further analyses (e.g. whole exome sequencing, comprehensive gene expression profiling) of the oviductal mouse HGSCs/MMMTs and comparison to human HGSCs will be needed to specifically address this issue.

A potential shortcoming of our model system is the development of non-oviductal tumours in a sizeable fraction of the 80 mice included in the study. This is probably related, at least in part, to the relatively long latency and time required for tumour progression, particularly in $B P R$ and $B P N$ mice. Our data suggest that this problem can be mitigated by focusing future work on TAM-treated BPP or BPRN mice with homozygous floxed (or in the case of Brca1, Brca1 ${ }^{\text {fl/del) }}$ alleles, which usually acquire STICs, HGSCs/MMMTs and metastatic disease before they develop non-oviductal tumours that necessitate early euthanasia.

Though the specific cell of origin of HGSC in humans remains unclear, our findings indicate that a population of Ovgp1-expressing cells in the oviduct is susceptible to transformation by somatic mutations akin to those commonly observed in human HGSCs. The transformed cells retain the capacity to differentiate along multiple Müllerian sub-lineages, both epithelial and mesenchymal. Notably, in contrast to the (Amhr2) Dicer-Pten double knock-out mice described by Kim et al., in which tumours initially arise in the oviductal stroma [17], the first recognizable lesions in our models are in the FTE. We have previously shown that like PAX8, OVGP1 is expressed primarily in secretory, rather than ciliated cells in the mouse FTE [22]. Not surprisingly, transformation of OVGP1-expressing cells results in tumours that express PAX8, a marker of secretory cells, but not tubulin, which is expressed by ciliated cells. The mucinous differentiation of oviductal epithelium noted in TAM-treated BPP mice was not seen in BPR, BPN or BPRN 
mice, suggesting a role for Pten inactivation in driving this metaplastic change. We have also observed squamous differentiation in occasional tumours arising in the context of Brca1, Trp53, and Pten inactivation (not shown). Mucinous and/or squamous differentiation is commonly seen in human endometrioid carcinomas, which have a high frequency of PTEN mutations [27]. We also note some differences between the oviductal lesions in our BPP mice and those based on transformation of PAX8-positive, rather than OVGP1-positive cells [18]. Although both models develop STICs and HGSCs that express PAX8, Perets et al. [18] did not observe carcinosarcomas or note the diffuse epithelial changes in the oviductal epithelium seen in our $B P P$ mice. These differences suggest, but do not prove, that the cells undergoing transformation in the two model systems are not identical. It is also possible that the tumour phenotypes in these two models is influenced by genetic background.

In summary, our GEMMs recapitulate many of the features seen in human HGSC. The mouse tumours are based on genetic alterations commonly observed in their human tumour counterparts, and arise in the FTE with a predilection for the oviductal equivalent of the human tubal fimbrial epithelium. The histologic features and biological behaviour are also similar to human HGSC. The models may prove to be particularly useful for studying the early phases of HGSC pathogenesis and effects of various factors associated with HGSC risk. For example, though women who have had multiple pregnancies or used oral contraceptives are welldocumented to have reduced risk of HGSC [28], the mechanisms by which this occurs remain unclear. Our GEMMs could be used to systematically test effects of these factors on tumour initiation and progression.

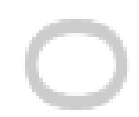

\section{Acknowledgements}

Research reported in this paper was supported by the National Cancer Institute of the National Institutes of Health under award numbers P30CA046592 (ERF and KRC) and R01CA188516 (KRC,YZ, RW, RK). 


\section{Author contribution statement}

The authors confirm that this manuscript describes original research, has not been previously published or presented in any form, and is not being considered for publication in any other scientific journal. All authors are aware of, and agree to, the content of the paper and accept authorship. YZ, RW, RK, ERF, and KRC conceived experiments and analyzed data. RW, $\mathrm{YZ}$, MSS, SS, and MG conceived and carried out experiments. All authors were involved in writing the paper and had final approval of the submitted version. 


\section{REFERENCES}

1. Bowtell DD, Bohm S, Ahmed AA, et al. Rethinking ovarian cancer II: reducing mortality from high-grade serous ovarian cancer. Nat Rev Cancer 2015; 15: 668-679.

2. Piek JM, van Diest PJ, Zweemer RP, et al. Dysplastic changes in prophylactically removed Fallopian tubes of women predisposed to developing ovarian cancer. J Pathol 2001; 195: 451456.

3. = Kindelberger DW, Lee Y, Miron A, et al. Intraepithelial carcinoma of the fimbria and pelvic serous carcinoma: Evidence for a causal relationship. Am J Surg Pathol 2007; 31: 161-169.

4. Kuhn E, Kurman RJ, Vang R, et al. TP53 mutations in serous tubal intraepithelial carcinoma and concurrent pelvic high-grade serous carcinoma--evidence supporting the clonal relationship of the two lesions. J Pathol 2012; 226: 421-426.

5. McDaniel AS, Stall JN, Hovelson DH, et al. Next-generation sequencing of tubal intraepithelial carcinomas. JAMA Oncology 2015; doi:10.1001/jamaoncol.2015.1618.

6. Eckert MA, Pan S, Hernandez KM, et al. Genomics of Ovarian Cancer Progression Reveals Diverse Metastatic Trajectories Including Intraepithelial Metastasis to the Fallopian Tube. Cancer Discov 2016.

7. Integrated genomic analyses of ovarian carcinoma. Nature 2011; 474: 609-615.

8. Patch AM, Christie EL, Etemadmoghadam D, et al. Whole-genome characterization of chemoresistant ovarian cancer. Nature 2015; 521: 489-494.

9. WHO Classification of Tumours of Female Reproductive Organs. (4th ed). International Agency for Research on Cancer: Lyon, 2014.

10. Brustmann H. Ovarian carcinosarcoma associated with bilateral tubal intraepithelial carcinoma: a case report. Int J Gynecol Pathol 2013; 32: 384-389.

11. Ardighieri L, Mori L, Conzadori S, et al. Identical TP53 mutations in pelvic carcinosarcomas and associated serous tubal intraepithelial carcinomas provide evidence of their clonal relationship. Virchows Arch 2016; 469: 61-69. 
12. Howell VM. Genetically engineered mouse models for epithelial ovarian cancer: are we there yet? Semin Cell Dev Biol 2014; 27: 106-117.

13. Szabova L, Yin C, Bupp S, et al. Perturbation of Rb, p53, and Brca1 or Brca2 cooperate in inducing metastatic serous epithelial ovarian cancer. Cancer Res 2012; 72: 4141-4153.

14. Connolly DC, Bao R, Nikitin AY, et al. Female Mice Chimeric for Expression of the Simian Virus 40 TAg under Control of the MISIIR Promoter Develop Epithelial Ovarian Cancer. Cancer Res 2003; 63: 1389-1397.

15. Flesken-Nikitin A, Choi KC, Eng JP, et al. Induction of carcinogenesis by concurrent inactivation of p53 and Rb1 in the mouse ovarian surface epithelium. Cancer Res 2003; 63: 3459-3463.

16. Flesken-Nikitin $\mathrm{A}$, Hwang $\mathrm{Cl}$, Cheng $\mathrm{CY}$, et al. Ovarian surface epithelium at the junction area contains a cancer-prone stem cell niche. Nature 2013; 495: 241-245.

17. Kim J, Coffey DM, Creighton CJ, et al. High-grade serous ovarian cancer arises from fallopian tube in a mouse model. Proc Natl Acad Sci U S A 2012.

18. Perets R, Wyant GA, Muto KW, et al. Transformation of the fallopian tube secretory epithelium leads to high-grade serous ovarian cancer in brca;tp53;pten models. Cancer Cell 2013; 24: 751765.

19. Arango NA, Kobayashi A, Wang Y, et al. A mesenchymal perspective of Mullerian duct differentiation and regression in Amhr2-lacZ mice. Mol Reprod Dev 2008; 75: 1154-1162.

20. Ordonez NG. Value of PAX 8 immunostaining in tumor diagnosis: a review and update. Advances in anatomic pathology 2012; 19: 140-151.

21. Miyoshi I, Takahashi K, Kon Y, et al. Mouse transgenic for murine oviduct-specific glycoprotein promoter-driven simian virus 40 large T-antigen: tumor formation and its hormonal regulation. Mol Reprod Dev 2002; 63: 168-176.

22. Wu R, Zhai Y, Kuick R, et al. Impact of oviductal versus ovarian epithelial cell of origin on ovarian endometrioid carcinoma phenotype in the mouse. J Pathol 2016; 240: 341-351.

23. Olive KP, Tuveson DA, Ruhe ZC, et al. Mutant p53 gain of function in two mouse models of LiFraumeni syndrome. Cell 2004; 119: 847-860. 
24. Anisimov VN, Ukraintseva SV, Yashin Al. Cancer in rodents: does it tell us about cancer in humans? Nat Rev Cancer 2005; 5: 807-819.

25. Son WC, Gopinath C. Early occurrence of spontaneous tumors in CD-1 mice and Sprague-Dawley rats. Toxicol Pathol 2004; 32: 371-374.

26. Reuber MD, Vlahakis G, Heston WE. Spontaneous hyperplastic and neoplastic lesions of the uterus in mice. J Gerontol 1981; 36: 663-673.

27. Obata K, Morland SJ, Watson RH, et al. Frequent PTEN/MMAC mutations in endometrioid but not serous or mucinous epithelial ovarian tumors. Cancer Res 1998; 58: 2095-2097.

28. Sopik V, Iqbal J, Rosen B, et al. Why have ovarian cancer mortality rates declined? Part I. Incidence. Gynecol Oncol 2015; 138: 741-749.

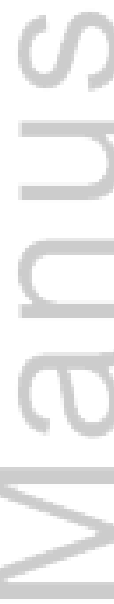

This article is protected by copyright. All rights reserved. 


\section{FIGURE LEGENDS}

Figure 1. Summary of oviductal tumour phenotype in TAM-treated Ovgp1-iCreER ${ }^{T 2}$ mice carrying various engineered Brca1, Rb1, Trp53, and Nf1 alleles. Data from all 80 mice are shown. Months post-TAM indicates the time points at which mice were euthanized. Where two time points are shown, the first represents months post-TAM at survival surgery (removal of right ovary and oviduct) and the second, months post-TAM at death. The diamond symbol $\diamond$ marks oviducts evaluated $\leq 6.5$ months post-TAM. STIC: serous tubal intraepithelial carcinoma; early HGSC: invasive HGSC confined to oviduct; HGSC: invasive HGSC extending beyond oviduct; MMMT: malignant mixed Müllerian tumour (carcinosarcoma).

Figure 2. Representative photomicrographs of STICs arising in BPRN mice. (A) Oviduct from mouse \#8141, showing STIC (boxed region) arising in portion of oviduct in close proximity to ovary. (B) Higher magnification of boxed region in A. (C) Oviduct from mouse \#8234, showing STIC (boxed region) in distal oviduct. (D) Higher magnification of boxed region in C. Scale bars represent $100 \mu \mathrm{m}$ unless otherwise indicated.

Figure 3. Representative photomicrographs of H\&E-stained sections showing progression of oviductal lesions in BPRN mice. STICs (panels $A$ and $E$ ), early HGSCs ( $B$, F), HGSC (C), MMMT (G) and metastases to omentum (D) and pancreas $(H)$ are shown. Scale bars represent $100 \mu \mathrm{m}$.

\section{Figure 4. STICs and HGSCs arising in BPRN mice express markers characteristic of} human HGSCs. Immunohistochemical staining for CK8, PAX8, Ki67 and p53 in representative STIC, early HGSC, HGSC, and metastatic HGSC is shown. Like normal oviductal epithelium, the tumour cells express CK8 and PAX8 - areas of CK8-expressing STIC and early HGSC are marked with blue arrows. The neoplastic cells have a high proliferative index, based on expression of Ki67. Overexpression of p53 is observed in occasional tumours with the Trp53 ${ }^{\text {LSL- }}$ ${ }^{R 172 H}$ allele (mouse \#3724), but not in mice carrying only $\operatorname{Trp}^{f 3^{f l}}$ alleles (\#3693, \#8234, and $\# 13604)$. Insets in selected panels show lesional epithelium at higher magnification. 
Figure 5. Representative HGSCs and MMMT identified in BPP mice at various times after TAM. Photomicrographs of H\&E and immunostained sections from oviductal STIC and early HGSC identified at 1 month (A-D), early HGSC at 2 months (E-H), and HGSC (I-L) and MMMT with metastasis to ovary (M-P) at 6 months post-TAM, as indicated. Oviduct in panel A shows both STIC (upper boxed area) and early HGSC (lower boxed area). Panels B, F, J, and N show higher magnification of lesions shown in panels $A, E, I$, and $M$. In panel $B$, the STIC is marked by blue arrowheads. Oviductal tumours arising in BPP mice uniformly express CK8 $(\mathrm{C}, \mathrm{G}, \mathrm{K}$, and $\mathrm{O})$ and PAX8 (D, H, L, and P).

Figure 6. Oviductal epithelium in BPP mice displays mucinous metaplasia not seen in $B P R, B P N$, or BPRN mice. Upper panels show normal mouse oviduct stained with H\&E (low and high magnification), PAS (periodic acid Schiff) and $A B$ (Alcian blue). Lower panels show comparably stained sections of oviduct from TAM-treated BPP mouse. Blue arrowheads point to accumulation of cytoplasmic mucin in the oviductal epithelium of BPP mouse, not seen in normal oviduct. The accumulated mucin is positive for both PAS (neutral mucin) and AB (acidic mucin). Note the paucity of PAS- or AB-positive mucin in normal oviductal epithelium. 
SUPPLEMENTARY MATERIAL ONLINE

Supplementary materials and methods NO

Supplementary figure legends YES

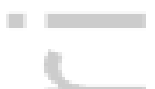

Figure S1. Metastatic HGSC to ovary in BPRN mouse (\#13576)

Figure S2. Representative photomicrographs of tubulin and PAX8 expression in STIC and HGSC

Figure S3. Cre-mediated gene recombination evaluated by PCR in genomic DNA isolated from representative matched mouse tails and oviductal tumours

Figure S4. Recombination status of engineered Rb1, Brca1, Trp53, and Nf1 alleles in DNA isolated from representative matched tails and non-oviductal tumours

Table S1. PCR primers for genotyping

Table S2. Summary of oviductal tumour phenotype and non-oviductal tumours in TAM-treated $B P R N, B P R$ and $B P N$ mice

Table S3. Summary of oviductal tumour phenotype in TAM-treated BPP mice 

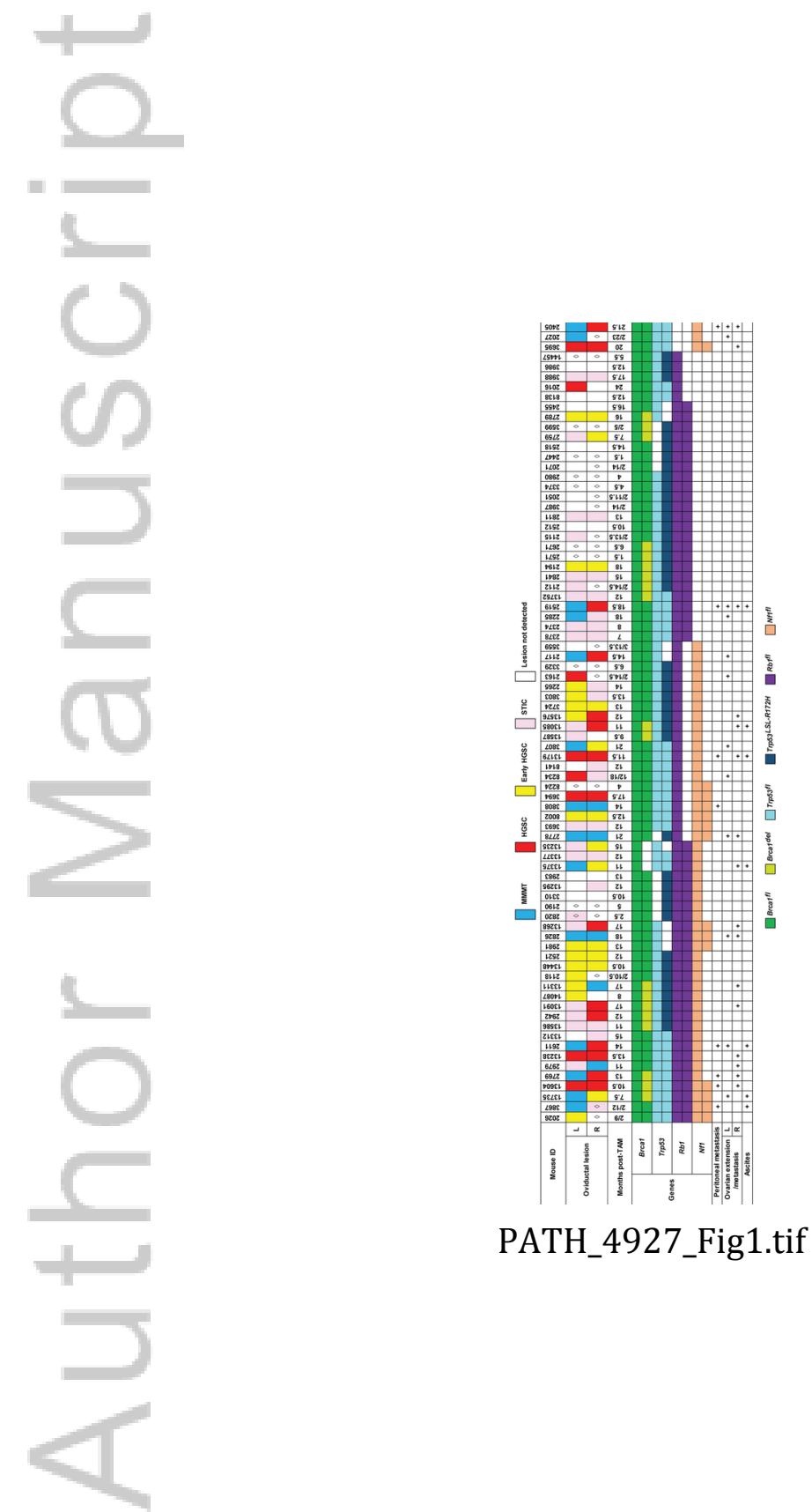

This article is protected by copyright. All rights reserved. 

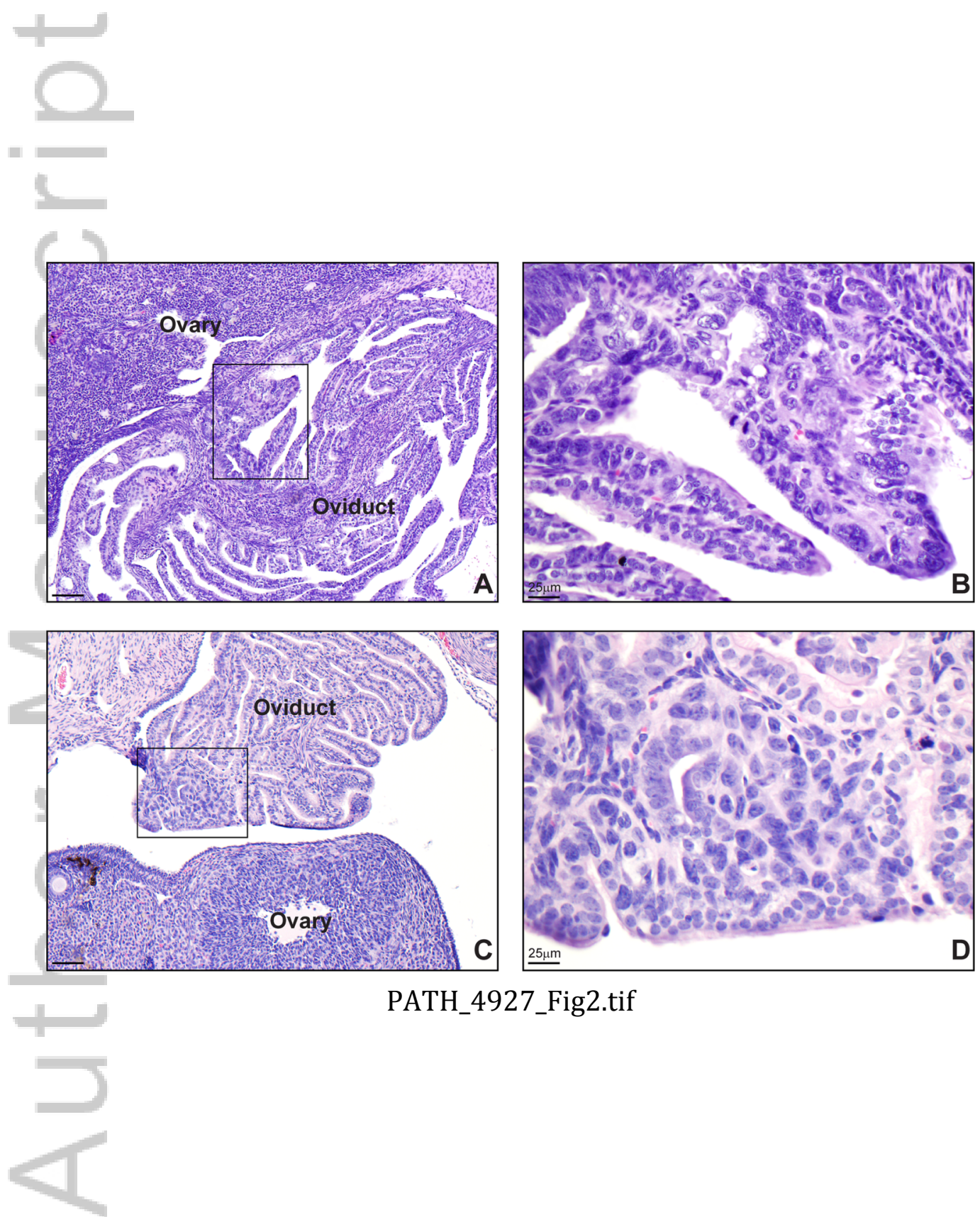

PATH_4927_Fig2.tif

This article is protected by copyright. All rights reserved. 


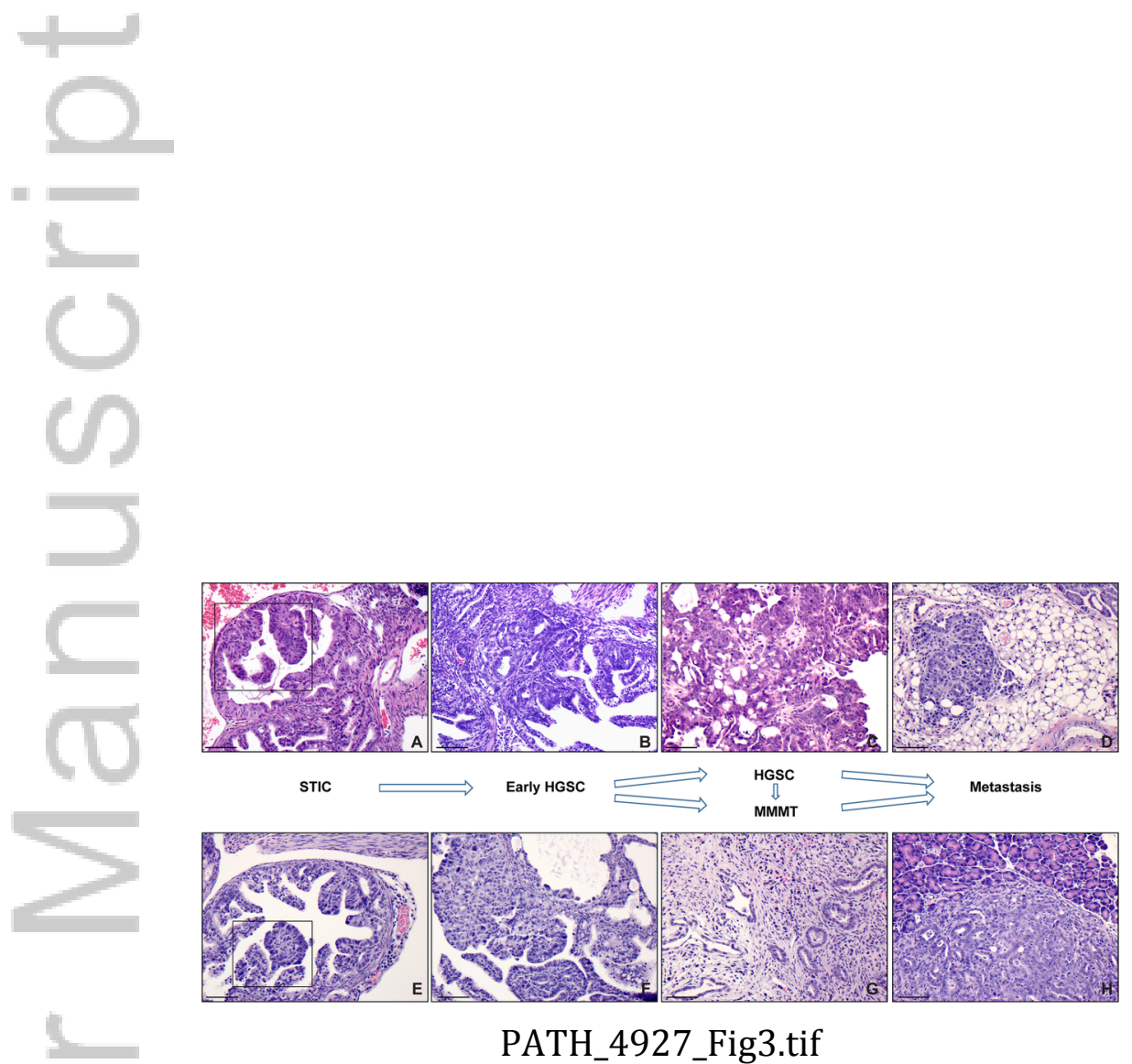

This article is protected by copyright. All rights reserved. 


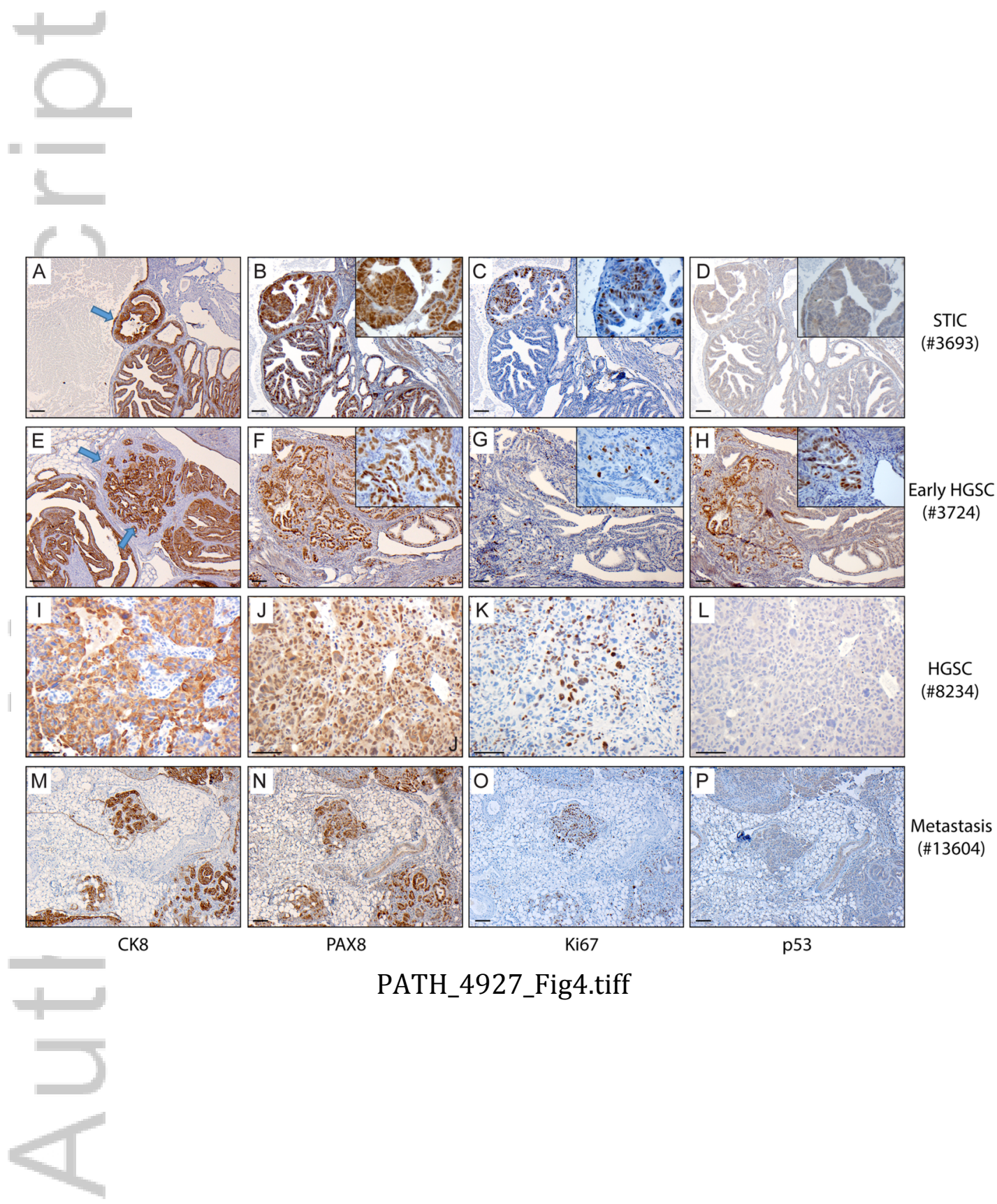

This article is protected by copyright. All rights reserved. 


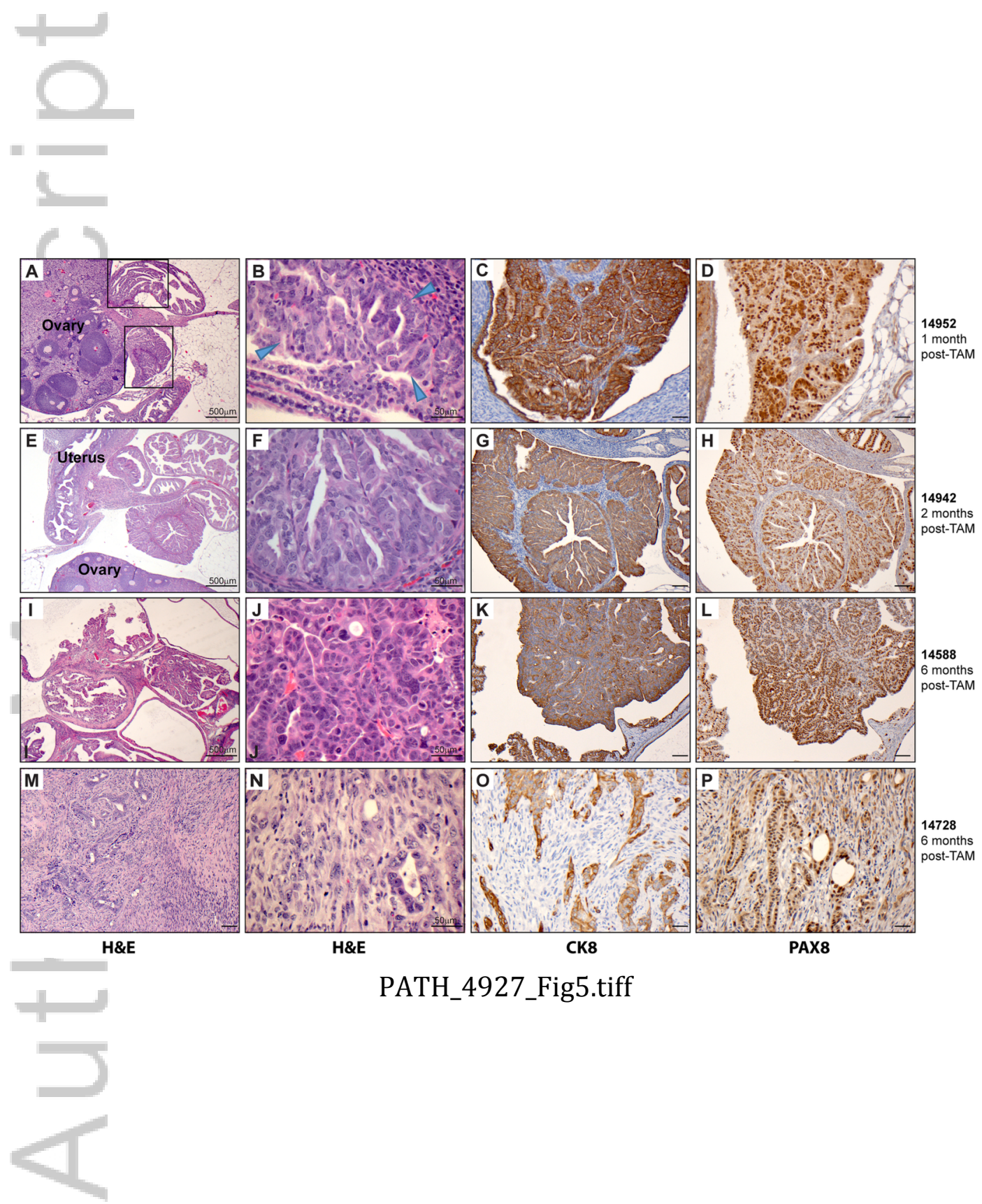

This article is protected by copyright. All rights reserved. 

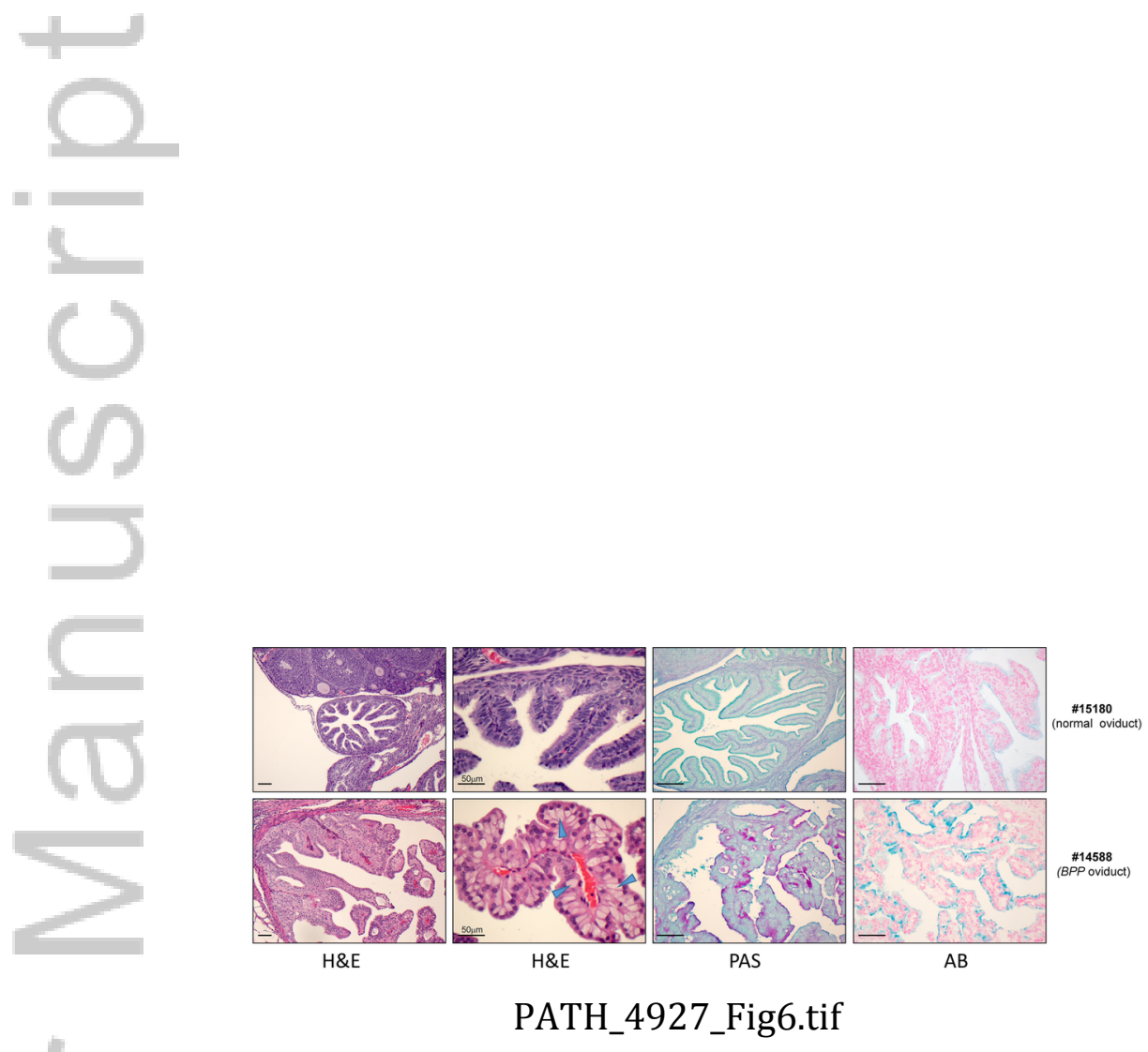

This article is protected by copyright. All rights reserved. 